\title{
Protein kinase A activates the Hippo pathway to modulate cell proliferation and differentiation
}

\author{
Fa-Xing Yu, ${ }^{1,2}$ Yifan Zhang, ${ }^{3,4}$ Hyun Woo Park, ${ }^{1,2}$ Jenna L. Jewell, ${ }^{1,2}$ Qian Chen, ${ }^{5}$ Yaoting Deng, ${ }^{3,4}$ \\ Duojia Pan, ${ }^{5}$ Susan S. Taylor, ${ }^{1,6}$ Zhi-Chun Lai, $^{3,4}$ and Kun-Liang Guan ${ }^{1,2,7}$ \\ ${ }^{1}$ Department of Pharmacology, ${ }^{2}$ Moores Cancer Center, University of California at San Diego, La Jolla, California 92093, USA; \\ ${ }^{3}$ Department of Biology, ${ }^{4}$ Department of Biochemistry and Molecular Biology, Intercollege Graduate Degree Program in \\ Genetics, The Pennsylvania State University, University Park, Pennsylvania 16802, USA; ${ }^{5}$ Department of Molecular Biology and \\ Genetics, Johns Hopkins University School of Medicine, Baltimore, Maryland 21205, USA; ${ }^{6}$ Department of Chemistry and \\ Biochemistry, University of California at San Diego, La Jolla, California 92093, USA
}

The Hippo tumor suppressor pathway plays an important role in tissue homeostasis that ensures development of functional organs at proper size. The YAP transcription coactivator is a major effector of the Hippo pathway and is phosphorylated and inactivated by the Hippo pathway kinases Lats1/2. It has recently been shown that YAP activity is regulated by G-protein-coupled receptor signaling. Here we demonstrate that cyclic adenosine monophosphate (cAMP), a second messenger downstream from $\mathrm{G \alpha}_{\mathrm{s}}$-coupled receptors, acts through protein kinase A (PKA) and Rho GTPases to stimulate Lats kinases and YAP phosphorylation. We also show that inactivation of YAP is crucial for PKA-induced adipogenesis. In addition, PKA activation in Drosophila inhibits the expression of Yorki (Yki, a YAP ortholog) target genes involved in cell proliferation and death. Taken together, our study demonstrates that Hippo-YAP is a key signaling branch of cAMP and PKA and reveals new insight into mechanisms of PKA in regulating a broad range of cellular functions.

[Keywords: Hippo; PKA; YAP; adipogenesis; proliferation]

Supplemental material is available for this article.

Received April 16, 2013; revised version accepted May 10, 2013.

The Hippo tumor suppressor pathway is fundamental in regulating cell proliferation, cell death, and cell differentiation; therefore, the tight control of this pathway and its cross-talk with other signaling pathways is critical for development and tissue homeostasis in adulthood (Yu and Guan 2013). Genetic manipulations of Hippo pathway components result in dramatic changes of organ size, and dysregulation of Hippo signaling is associated with tumorigenesis, demonstrating the critical function of this pathway in both physiological tissue growth control and pathological conditions (Yu and Guan 2013). Core components of the Hippo pathway were first established in Drosophila and later shown to be highly conserved in mammals (Justice et al. 1995; Xu et al. 1995; Kango-Singh et al. 2002; Tapon et al. 2002; Harvey et al. 2003; Jia et al. 2003; Pantalacci et al. 2003; Udan et al. 2003; Wu et al. 2003; Huang et al. 2005; Lai et al. 2005). Core components of the mammalian Hippo pathway contain a kinase cascade of Mst1/2 (Hippo) and Lats1/2. Mst1/2 in complex

${ }^{7}$ Corresponding author

E-mail kuguan@ucsd.edu

Article is online at http://www.genesdev.org/cgi/doi/10.1101/gad.219402.113. with the scaffold Sav1 phosphorylates and activates Lats $1 / 2$ kinases. Activated Lats1/2 in complex with its regulatory protein, Mob, in turn phosphorylates and inhibits YAP and TAZ, two homologous transcription coactivators (Wu et al. 2003; Chan et al. 2005; Huang et al. 2005; Callus et al. 2006; Dong et al. 2007; Zhao et al. 2007; Lei et al. 2008; Oh and Irvine 2008). YAP/TAZ are major downstream effectors of the Hippo pathway, responsible for the expression of a large number of genes important for cell proliferation and survival (Goulev et al. 2008; Wu et al. 2008; Zhang et al. 2008; Zhao et al. 2008). Phosphorylation of YAP/TAZ by the Lats kinases results in their cytoplasmic retention and ubiquitin-mediated degradation, which ultimately leads to inhibition of YAP/TAZ (Kanai et al. 2000; Dong et al. 2007; Zhao et al. 2007, 2010; Lei et al. 2008; Oh and Irvine 2008; Liu et al. 2010; Ren et al. 2010).

The Hippo pathway has been implicated in cell contact inhibition, as YAP/TAZ display a dramatic cell densitydependent subcellular localization and phosphorylation (Zhao et al. 2007). In addition, mechanic stress has also been shown to modulate YAP/TAZ activity (Dupont et al. 2011; Wada et al. 2011; Zhao et al. 2012). Recently, we demonstrated that extracellular diffusible signals modulate 
the Hippo pathway through G-protein-coupled receptor (GPCR) signaling (Mo et al. 2012; Yu et al. 2012a,b). GPCR is the largest family of cell surface receptors encoded in the human genome and has been implicated in almost every aspect of physiological regulation. We observed that hormonal factors like LPA, S1P, and Thrombin can activate $\mathrm{G} \alpha_{12 / 13}$ to stimulate YAP/TAZ, which mediate the effect of these signals on gene expression, cell proliferation, and migration (Mo et al. 2012; Yu et al. 2012b). Similar observations were also reported by $\mathrm{Wu}$ and colleagues (Miller et al. 2012). In contrast, ligands of $\mathrm{G}_{\mathrm{s}}$-coupled receptors, such as epinephrine and glucagon, stimulate Lats1/2 and result in inhibition of YAP/TAZ (Yu et al. $2012 \mathrm{~b}$ ). These findings suggest that the activity of YAP/ TAZ can be positively or negatively modulated by a wide range of extracellular signals via GPCRs in a manner dependent on which intracellular signaling pathway is stimulated.

Activation of $\mathrm{G}_{\mathrm{s}}$-coupled receptors usually results in accumulation of cyclic adenosine monophosphate (cAMP), an important second messenger with diverse physiological functions, including cell proliferation and differentiation (Cho-Chung 1990). Despite extensive studies, the precise molecular mechanisms of how cAMP regulates cell proliferation and differentiation is not fully understood (Stork and Schmitt 2002). In this study, we demonstrate that cAMP acts through protein kinase A (PKA, cAMP-dependent protein kinase) and Rho GTPases to stimulate Lats kinase activity and inhibit YAP/TAZ. Inhibition of YAP/TAZ is critical for cAMP and PKA to promote adipogenesis and suppress growth, establishing Hippo-YAP as a signaling branch downstream from cAMP and PKA.

\section{Results}

\section{cAMP signaling stimulates YAP phosphorylation}

Activation of $\mathrm{G}_{\mathrm{s}}$-coupled receptors can stimulate adenylyl cyclase (AC) and result in an increase of cAMP production (Sassone-Corsi 2012). We treated MDA-MB-231 breast cancer cells with epinephrine, a ligand for $\beta 2$ adrenergic receptor that increases cAMP (Supplemental Fig. S1). As anticipated, we observed a transient induction of phosphorylation of the cAMP response element-binding protein (CREB), a direct target of PKA and an indicator of cAMP accumulation and PKA activation (Fig. 1A). Interestingly, YAP phosphorylation was also transiently increased in response to epinephrine, as assessed by a phospho-specific antibody against Ser127, which is a direct Lats phosphorylation site responsible for cytoplasmic localization, or a phos-tag gel, which resolves YAP protein based on phosphorylation status (Fig. 1A). When cellular cAMP was induced by forskolin, a pharmacological activator of AC (Supplemental Fig. S1), phosphorylation of both YAP and CREB was similarly induced, as seen with epinephrine treatment (Fig. 1B). The phosphorylation of YAP in response to cAMP was maximum at $1 \mathrm{~h}$ and started to decline at $4 \mathrm{~h}$. Notably, the response of CREB to CAMP singling was swifter (Fig. 1C), suggesting that YAP and CREB might be
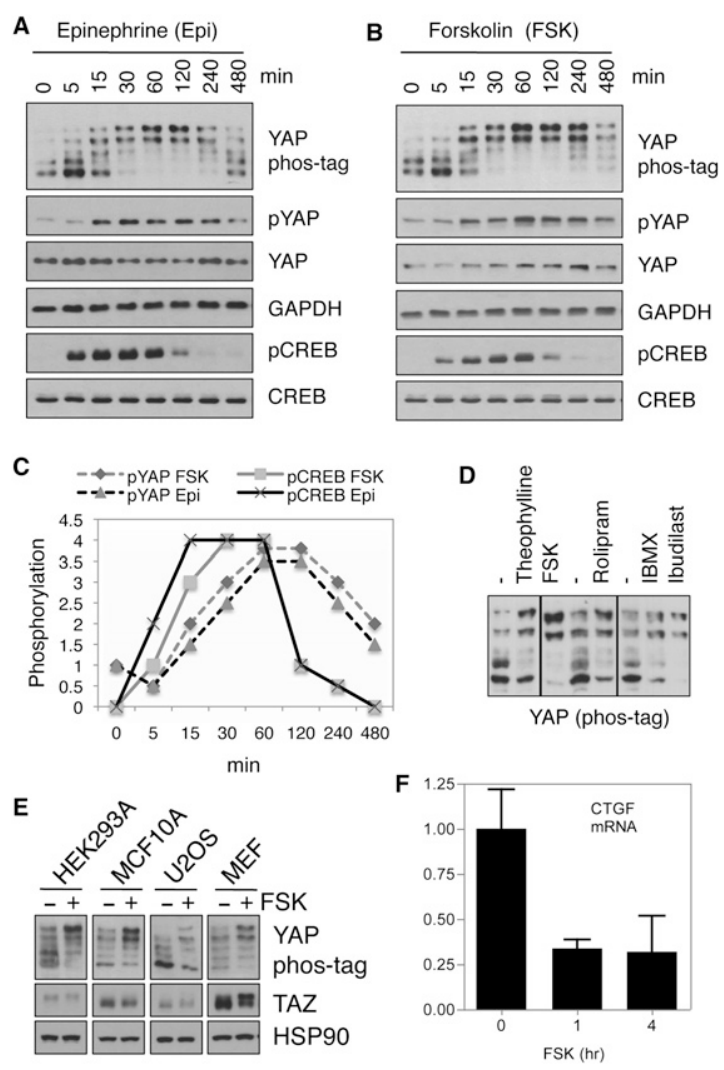

Figure 1. CAMP signaling induces YAP phosphorylation and inactivation. $(A, B)$ MDA-MB-231 cells were treated with $10 \mu \mathrm{M}$ epinephrine $(A)$ or forskolin $(B)$ for the indicated durations, and cell lysates were subjected to immunoblotting using the indicated antibodies. $(C)$ Time course of YAP and CREB phosphorylation in response to epinephrine or forskolin (the value for time 0 was arbitrarily set). (D) MDA-MB-231 cells were treated with different PDE inhibitors-ibudilast (100 $\mu \mathrm{M})$, IBMX $(100 \mu \mathrm{M})$, rolipram (50 $\mu \mathrm{M})$, or theophylline $(1 \mathrm{mM})$ - for $1 \mathrm{~h}$, and the phosphorylation status of YAP was determined by phos-tag gels. (E) HEK293A, MCF10A, U2OS, or MEF cells were treated with or without 10 $\mu \mathrm{M}$ forskolin for $1 \mathrm{~h}$. YAP phosphorylation was assessed using phos-tag gels, and the same lysates were also used to blot for TAZ protein levels. $(F)$ MCF10A cells were serum-starved overnight and treated with $10 \mu \mathrm{M}$ forskolin for 1 or $4 \mathrm{~h}$, mRNA was extracted, and the expression level of CTGF was determined using real-time RT-PCR.

regulated by different molecular mechanisms downstream from cAMP (see below).

Intracellular cAMP levels are controlled by both biosynthesis and degradation. In mammalian cells, multiple phosphodiesterases (PDEs) are able to break down cAMP (Supplemental Fig. S1). Many pharmaceutical drugs are direct PDE inhibitors that can be used to increase cellular cAMP levels (Supplemental Fig. S1; Sassone-Corsi 2012). Several nonselective PDE inhibitors (theophylline, IBMX, and ibudilast) and PDE4 selective inhibitors (rolipram) all induced YAP phosphorylation (Fig. 1D), further supporting the role of cAMP in stimulating YAP phosphorylation. In addition, these data also suggest that PDE inhibitors might be useful tools for restricting YAP activity. 
We tested the effect of forskolin on YAP phosphorylation in multiple cell lines, including U2OS, MCF10A, HEK293A, and mouse embryonic fibroblasts (MEFs). In all cases, YAP phosphorylation was increased by forskolin treatment (Fig. 1E). TAZ is a YAP homolog similarly regulated by the Hippo pathway (Lei et al. 2008). As expected, TAZ phosphorylation was increased, as indicated by the decreased electrophoretic mobility (Fig. 1E). Moreover, TAZ protein levels were modestly reduced in forskolin-treated cells because TAZ is destabilized by phosphorylation (Liu et al. 2010). These results indicate that the cross-talk between cAMP signaling and the Hippo pathway is a conserved phenomenon in different cell types, and both Hippo pathway effectors, YAP and TAZ, are inactivated by cAMP.

YAP/TAZ are transcriptional coactivators. To determine the functional significance of intracellular cAMP on YAP activity, we determined expression of YAP/TAZ target genes. Indeed, the expression of CTGF, which is a direct YAP/TAZ target gene, was inhibited by forskolin in MCF10A cells (Fig. 1F), further supporting the idea that cAMP inhibits YAP and TAZ activity.

\section{cAMP signals through PKA to stimulate YAP phosphorylation}

Exchange protein activated by cAMP (Epac) and PKA are two downstream effectors mediating most physiological functions of cAMP (Supplemental Fig. S1). Epac proteins and the regulatory $(\mathrm{R})$ subunits of PKA contain cAMPbinding domains that function as cAMP sensors (Gloerich and Bos 2010; Taylor et al. 2012). We investigated whether PKA or Epac signaling mediated the effect of cAMP on YAP phosphorylation. Overexpression of the catalytic (C) subunit $\alpha$ (PRKACA) induced YAP phosphorylation, whereas overexpression of the PKA kinasedead mutant decreased YAP phosphorylation (Fig. 2A). In contrast, overexpression of wild-type or constitutively active Rap1b, an effector of Epac (Gloerich and Bos 2010), did not show a significant effect on YAP phosphorylation (Fig. 2B). These results indicate that PKA rather than Epac mediates the effect of cAMP on YAP inhibition.

PKA C subunits form a complex with $\mathrm{R}$ subunits under basal state, and the kinase activity is restricted; cAMP binding to the $\mathrm{R}$ subunits induces a conformational change and releases the $\mathrm{C}$ subunits and therefore results in PKA kinase activation (Supplemental Fig. S1; Taylor et al. 2012). To study the involvement of PKA on YAP inactivation, we employed mutant PKA R subunits that interact with PKA $C$ subunits in a manner unresponsive to cAMP. When mutant PKA R subunits (RI $\alpha$ and RII $\alpha$ ) were overexpressed, forskolin-induced YAP phosphorylation was completely blocked (Fig. 2C). We also used shRNA to knock down the PKA C subunits, and when PKA C $\alpha$ expression was down-regulated, the induction of YAP phosphorylation by forskolin or epinephrine was strongly compromised (Fig. 2D). Moreover, when cells were treated with a PKA inhibitor, KT5720 (Supplemental Fig. S1), YAP phosphorylation was decreased, and the effect of forskolin and epinephrine was largely blocked by KT5720 (Fig. 2E). We also examined primary hepatocytes isolated from mice. Stimulation with glucagon, a ligand known to activate PKA, increased YAP phosphorylation (Fig. 2F). Similar to that of epinephrine, the PKA inhibitor KT5720 abolished the effect of glucagon on YAP phosphorylation (Fig. 2F). Collectively, these data establish that PKA is the key mediator of cAMP in stimulating YAP phosphorylation.
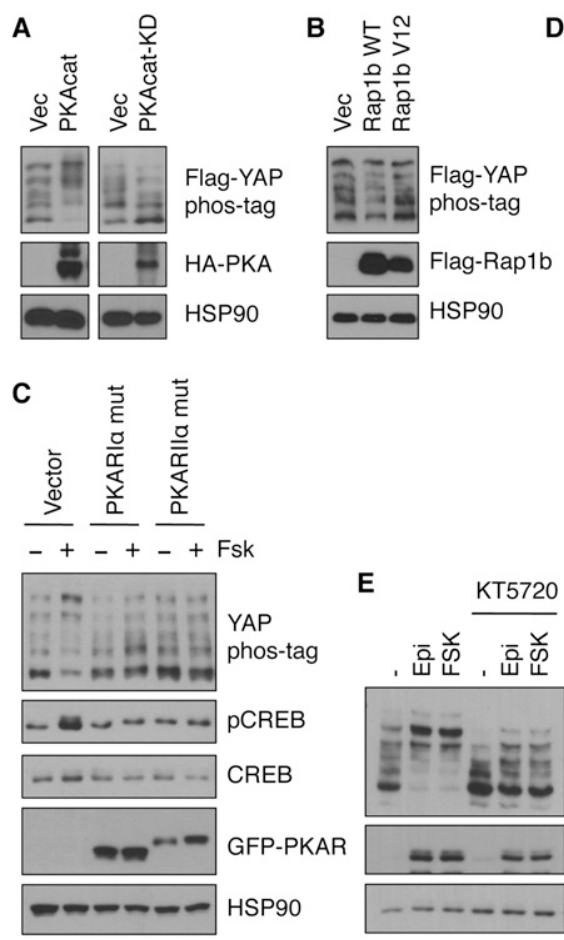

E

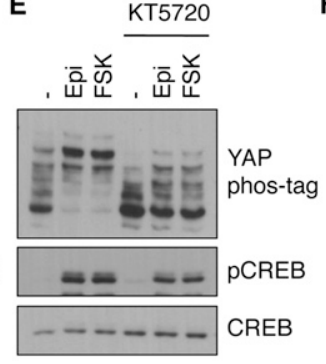

Control PKA\#1 PKA\#2 shRNA
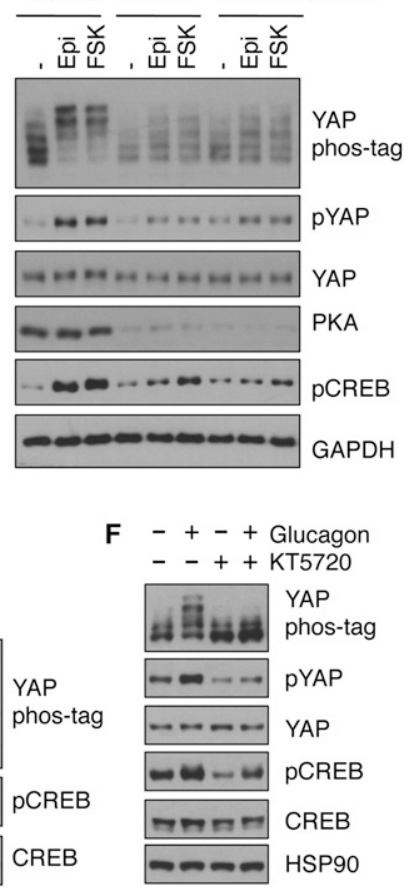

Figure 2. cAMP signaling to YAP phosphorylation is mediated by PKA. (A) Flag-YAP was cotransfected with or without HA-tagged wild-type or kinase-dead PKA catalytic subunit; after $24 \mathrm{~h}$, cell lysates were prepared, and phosphorylation of Flag-YAP was determined. $(B)$ Similar to $A$ except that Flag-tagged wild-type or constitutively active Rap1b was transfected. (C) HEK293A cells were transfected with mutant PKA regulatory subunits (PKARI $\alpha$ or PKARII $\alpha$ ); after $16 \mathrm{~h}$, cells were treated with or without $10 \mu \mathrm{M}$ forskolin for $1 \mathrm{~h}$, and YAP or CREB phosphorylation was assessed. $(D)$ Stable cell lines (MDAMB-231) expressing control shRNA or shRNAs targeting the PKA catalytic subunit ( $\alpha$ isoform) were established and treated with or without $10 \mu \mathrm{M}$ epinephrine or forskolin for $1 \mathrm{~h}$. Cell lysates were subjected to immunoblotting to determine the level of YAP and CREB phosphorylation. (E) MDA-MB-231 cells were pretreated with or without PKA inhibitor KT5720 (5 $\mu \mathrm{M})$ for $30 \mathrm{~min}$ and then stimulated with $10 \mu \mathrm{M}$ epinephrine or forskolin for $1 \mathrm{~h}$; YAP and CREB phosphorylation was then determined. $(F)$ Similar to $E$ except that primary hepatocytes were used, and glucagon was used to induce PKA activity. 
Yu et al.

cAMP stimulates Lats kinases to induce YAP phosphorylation

YAP is phosphorylated by the Lats $1 / 2$ kinases on five serine residues within the HXRXXS motifs, including S127, and can be phosphorylated on additional sites by other kinases (Zhao et al. 2010). To determine whether cAMP regulates the Lats phosphorylation sites in YAP, a 5SA mutant YAP (with all five Lats targeting sites mutated to alanine) was transfected into cells and then treated with or without forskolin. Forskolin failed to induce a significant change in phosphorylation of the YAP-5SA mutant, as assessed by phos-tag gel (Fig. 3A), suggesting that PKA likely acts through the Hippo pathway kinases to stimulate YAP phosphorylation. To test the function of MST kinases in CAMP-induced YAP phosphorylation, MST1/2 expression was down-regulated

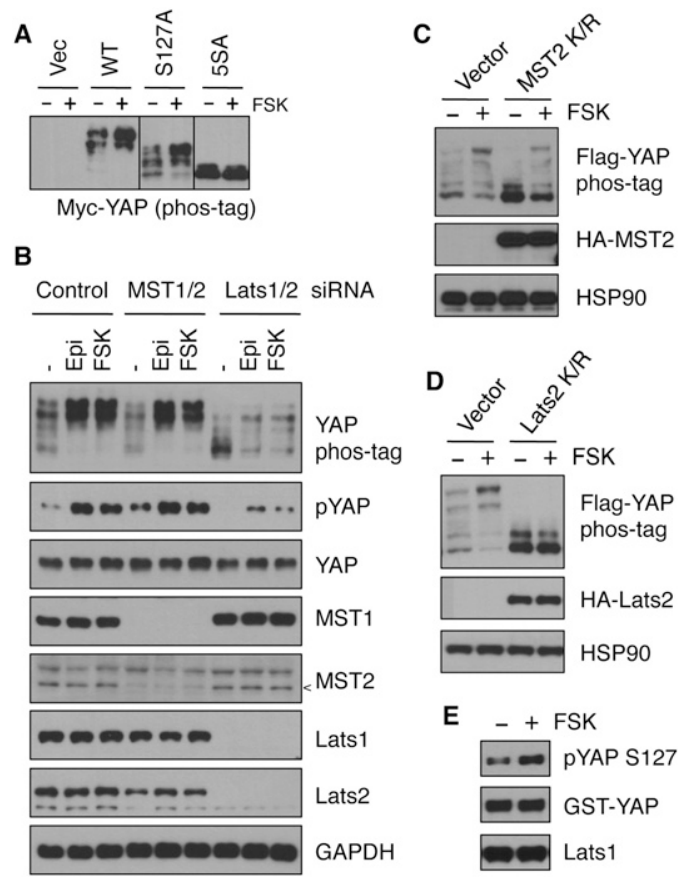

Figure 3. PKA increases YAP phosphorylation by stimulating kinase activity of Lats1/2. (A) Myc-tagged wild-type or S127A or 5SA mutant YAP were transfected into HEK293A cells, and, after $16 \mathrm{~h}$, cells were treated with or without $10 \mu \mathrm{M}$ forskolin for $1 \mathrm{~h}$. YAP phosphorylation was assessed by phos-tag gel. (B) MDA-MB-231 cells were transfected with control, MST1/2, or Lats $1 / 2$ siRNAs. Two days later, cells were treated with $10 \mu \mathrm{M}$ epinephrine or forskolin for $1 \mathrm{~h}$. Cell lysates were subjected to immunoblotting to assess knockdown efficiency and YAP phosphorylation. The arrowhead indicates MST2 position. $(C, D)$ Flag-YAP was cotransfected into HEK293A cells with or without K/R mutants (kinase-dead) of MST2 $(C)$ or Lats2 $(D)$; after $16 \mathrm{~h}$, cells were stimulated with $10 \mu \mathrm{M}$ forskolin for $1 \mathrm{~h}$, and phos-tag gels were used to determine the phosphorylation status of Flag-YAP. (E) MDA-MB-231 cells were untreated or treated with $10 \mu \mathrm{M}$ forskolin for $1 \mathrm{~h}$, endogenous Lats1 was immunoprecipitated and subjected to kinase assay using GSTYAP as substrate, and phosphorylation of GST-YAP by Lats1 was monitored by YAP phosphorylation at S127. by siRNAs, and the phosphorylation status of YAP was relatively normal in response to forskolin treatment (Fig. 3B). Overexpression of MST2 K/R (lysine mutated to arginine), a kinase-dead mutant, resulted in a lower basal YAP phosphorylation. However, under this condition, forskolin was still capable of inducing YAP phosphorylation (Fig. 3C), suggesting that MST may not be involved in cAMP response. In contrast, when Lats $1 / 2$ expression was down-regulated by siRNAs, the basal YAP phosphorylation was lower, and, notably, forskolininduced YAP phosphorylation was significantly impaired (Fig. 3B). When Lats2 K/R, a kinase-dead mutant, was overexpressed, the basal YAP phosphorylation was reduced, and, importantly, the effect of forskolin on YAP phosphorylation was abolished (Fig. 3D). These data indicate that Lats is involved in YAP phosphorylation in response to cAMP.

We next determined whether cAMP could increase Lats kinase activity. Lats1 kinase was immunoprecipitated from cells treated with or without forskolin, and in vitro Lats kinase activity was measured using purified GST-YAP as a substrate. Our results indicate that Lats kinase activity is indeed induced by forskolin (Fig. 3E). Collectively, the above results reveal that Lats kinases are required for cAMP and PKA to induce YAP phosphorylation.

\section{Rho GTPases are required for PKA to modulate YAP phosphorylation}

The response of YAP phosphorylation to cAMP is slower than that of CREB phosphorylation (Fig. 1C), suggesting that PKA may not directly phosphorylate a core component of the Hippo pathway. Consistently, we could not activate Lats1 in vitro using purified PKA (data not shown). Recently, it has been reported that Rho GTPases can regulate the Hippo pathway and plays a major role from $\mathrm{G} \alpha_{12 / 13}$-coupled receptors to YAP phosphorylation (Dupont et al. 2011; Miller et al. 2012; Mo et al. 2012; Yu et al. 2012b; Zhao et al. 2012). Interestingly, PKA has been shown to modulate the actin cytoskeleton by inhibition of RhoA, which is achieved by phosphorylation of RhoA, Rho GDP dissociation inhibitor (RhoGDI), or Rho guanine nucleotide exchange factors (RhoGEFs) (Qiao et al. 2008; Meiri et al. 2009; Tkachenko et al. 2011). We hypothesized that PKA might induce Lats $1 / 2$ activity by repressing RhoA. Indeed, the phosphorylation of myosin light chain 2 (MLC2), a target of Rho-associated protein kinase (ROCK), was reduced when cells were treated with forskolin (Fig. 4A), indicating a decreased RhoA activity when PKA is activated. When cells were transfected with wild-type or constitutively active RhoA, forskolin was unable to induce YAP phosphorylation (Fig. 4B). Complementarily, when RhoGDI, an inhibitor of Rho GTPases, was overexpressed, the PKA inhibitor KT5720 was unable to induce YAP/TAZ dephosphorylation (Fig. 4C). Taken together, these observations suggest that RhoA is a major mediator for cAMP or PKA to regulate YAP phosphorylation, and we propose that PKA increases Lats $1 / 2$ activity and YAP phosphorylation by inhibiting Rho GTPases. 
A

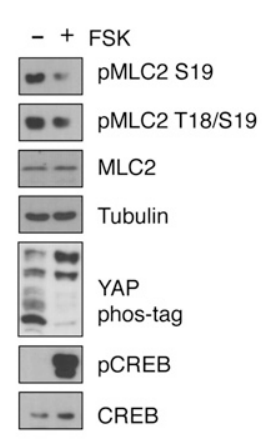

B

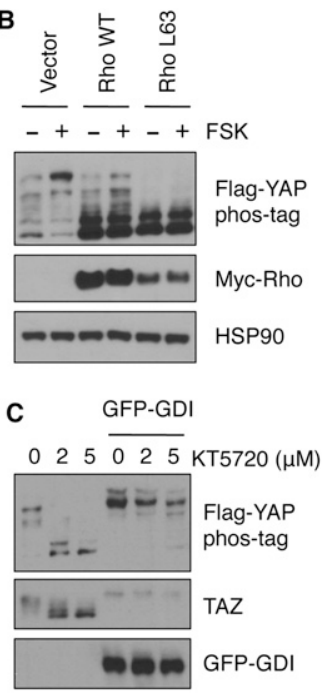

Figure 4. Rho GTPases mediate the effect of PKA on YAP phosphorylation. (A) MDA-MB-231 cells were treated with $10 \mu \mathrm{M}$ forskolin for $1 \mathrm{~h}$, and cell lysates were subjected to immunoblotting. Phosphorylation of MLC2, CREB, and YAP was determined. (B) Flag-YAP was cotransfected into HEK293A cells with wild-type or constitutively active RhoA, and, after $16 \mathrm{~h}$, cells were stimulated with $10 \mu \mathrm{M}$ forskolin for $1 \mathrm{~h}$ before Western blotting. (C) Flag-YAP was cotransfected into HEK293A cells with or without GFP-tagged RhoGDI, and, after $16 \mathrm{~h}$ of incubation in serum-free medium, cells were treated with or without KT5720 for $1 \mathrm{~h}$. Phosphorylation of Flag-YAP and endogenous TAZ was determined.

Hippo pathway activation is required for cAMPor PKA-induced adipogenesis

PKA and cAMP play important roles in cell lineage specification during metazoan development (Lane and Kalderon 1993). For instance, PKA has been shown to promote adipogenesis (Rosen and MacDougald 2006), although molecular mechanisms underlying PKA-regulated cell differentiation are not fully understood. Notably, TAZ displays activity opposite to PKA and can inhibit adipogenesis (Hong et al. 2005). As shown above, YAP and TAZ are negatively regulated by PKA; therefore, the Hippo pathway might function downstream from PKA in regulating cell differentiation.

To test the above hypothesis, the effect of PKA or the Hippo pathway on adipocyte differentiation of murine fibroblast 3T3-L1 cells was examined. We found that YAP phosphorylation was repressed by KT5720 and induced by forskolin or IBMX in 3T3-L1 cells (Fig. 5A). TAZ protein level was increased by KT5720 and decreased by forskolin or IBMX (Fig. 5A), consistent with TAZ degradation upon phosphorylation (Liu et al. 2010). Adipogenesis was initiated by addition of insulin, dexamethasone, and troglitazone (Tro) with or without PKA activator or inhibitor. The formation of lipid droplets was visualized by oil red staining. As expected, IBMX induced, whereas KT5720 inhibited, adipogenesis, indicating a stimulatory role of PKA in adipocyte differentiation (Fig. 5B). Next, we examined the effect of YAP and TAZ on adipogenesis and

found that knockdown of YAP and TAZ promoted adipogenesis, an effect similar to IBMX treatment (Fig. 5C,D). Interestingly, knockdown of YAP and TAZ also largely blocked the effect of the PKA inhibitor KT5720 (Fig. 5E). On the other hand, YAP overexpression strongly inhibited the ability of IBMX or forskolin to induce adipogenesis (Fig. 5F). Furthermore, the expression of multiple adipogenesis markers was abolished by YAP overexpression even in the presence of IBMX or forskolin (Fig. 5G). Therefore, YAP/TAZ activation is required for the PKA inhibitor to suppress adipocyte differentiation, whereas YAP/TAZ inhibition is crucial for IBMX to induce the differentiation program. Taken together, our data support a model in which modulation of YAP and TAZ activity is required for PKA signaling to regulate adipogenesis.

\section{Inactivation of Yorki (Yki) by PKA in Drosophila}

We next investigated whether the Drosophila YAP ortho$\log$ (Yki) is similarly regulated by PKA. In Drosophila $\mathrm{S} \mathrm{R}^{+}$cells, when the Drosophila PKA ortholog (PKA-C1) was knocked down by dsRNA (RNAi), the Yki transcription activity was significantly increased, as assessed by a luciferase assay (Fig. 6A). Therefore, the cross-talk between PKA and the Hippo pathway might be conserved in Drosophila. During Drosophila imaginal disc development, PKA-C1 has been shown to be a potent growth inhibitor, and loss of PKA function leads to ectopic limb (such as wing) formation (Jiang and Struhl 1995; Lepage et al. 1995; Li et al. 1995; Pan and Rubin 1995). To test the possibility that PKA-C1 might regulate Hpo signaling for growth control, expression of several Yki target genes, including expanded (ex), Cyclin E (CycE), and Diap1, was determined at the transcript level. In larval wing discs, loss of PKA-Cl activity caused an increase of expression of $e x, C y c E$, and Diap1 (Fig. 6B). On the contrary, overexpression of PKA-C1 resulted in a moderate yet significant reduction of expression of these genes (Fig. 6B). Moreover, PKA-C1 overexpression induced Yki phosphorylation (Fig. 6C) and was sufficient to reduce the level of Diap1 protein and promote programmed cell death, as revealed by increased caspase 3 staining (Fig. 6D-G). These results suggest that PKA inhibits Yki activity in developing tissues to restrict proliferation and promote apoptosis.

\section{Discussion}

\section{The signaling relay from cAMP to the Hippo-YAP} pathway

In this study, we show that cAMP acts through PKA to stimulate Lats kinase activity and YAP phosphorylation, and the Rho GTPases likely mediate the effect of PKA to Hippo-YAP regulation (Fig. 7). Although the Hippo homolog MST1/2 may not be involved in YAP regulation in response to CAMP, we would still prefer to retain the name of Hippo given the fact that YAP/TAZ are the only known major functional output of the Hippo pathway. Our data establish Hippo-YAP as a physiologically relevant signaling branch downstream from PKA. The precise 
A

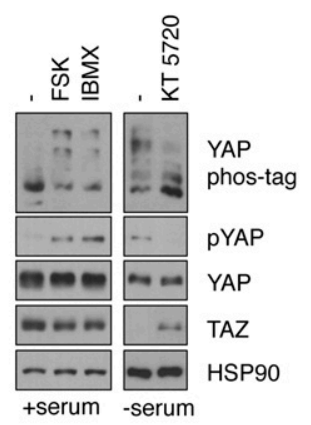

B

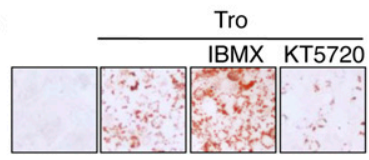

G

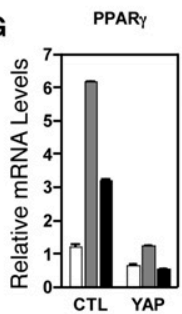

C
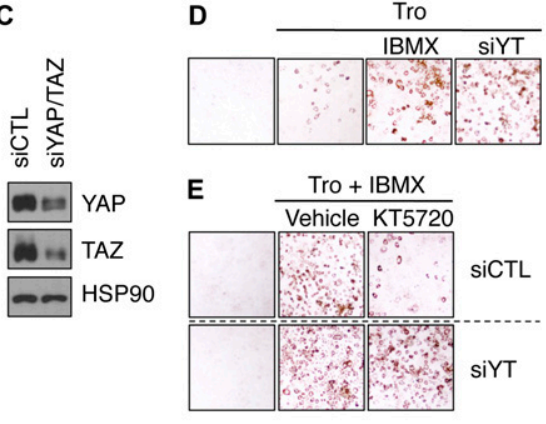

$\mathbf{F}$

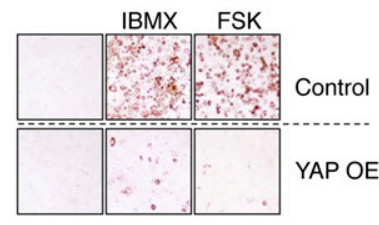

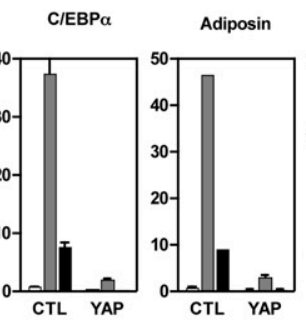
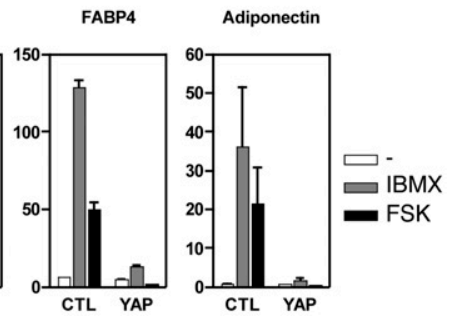

Figure 5. YAP/TAZ mediate the effect of cAMP in adipogenesis. (A) 3T3-L1 cells were treated with $10 \mu \mathrm{M}$ forskolin or $100 \mu \mathrm{M}$ IBMX for $1 \mathrm{~h}$, or serum-starved 3T3-L1 cells were treated with $5 \mu$ M KT5720 for $1 \mathrm{~h}$, and YAP phosphorylation and TAZ protein levels were determined. (B) 3T3-L1 cells were incubated under adipocyte differentiation conditions with IBMX or KT5720. (Tro) Troglitazone. IBMX increased, whereas KT5720 repressed, adipogenesis, as assessed by oil red staining. $(C, D)$ 3T3-L1 cells were transfected with control or YAP and TAZ siRNAs (siYT), and the knockdown efficiency was determined by immunoblotting (shown in $C$ ). $(D)$ These cells are also subjected to adipogenesis. (E) 3T3-L1 cells were transfected with control or YAP and TAZ siRNAs. Cells were treated with Tro and IBMX in the presence of vehicle (DMSO) or KT5720 as indicated. Adipocyte differentiation was measured by oil red staining. $(F)$ Overexpression of YAP abolished IBMX- or forskolin-induced adipogenesis. $(G)$ Following differentiation (as in $F$ ), cells were lysed, and the expression of adipogenesis marker genes was determined by real-time RT-PCR; the mRNA level was normalized to that of cells incubated in growth medium. molecular mechanism connecting Rho to Lats kinase requires further investigation.

The kinase activity of endogenous Lats1 (Fig. 3E) and overexpressed Lats2 (data not shown) is increased upon PKA activation, and the effect of PKA activation on YAP phosphorylation is blocked by a kinase-dead mutant Lats2, suggesting that the effect of PKA on YAP phosphorylation is mediated by Lats kinases. In contrast, MST1/2 are not required for PKA-induced YAP phosphorylation because the effect of forskolin and epinephrine on YAP phosphorylation is intact when both MST1 and MST2 are down-regulated by siRNA (Fig. 3B), and expression of kinase-dead mutant MST2 does not block the effect of forskolin on YAP phosphorylation (Fig. 3C). Consistently, MST1 kinase activity and phosphorylation at the activation loop are not modulated by forskolin treatment (Yu et al. 2012b). However, phosphorylation of the hydrophobic motif of Lats1 is induced by cAMP signaling (Yu et al. 2012b), indicating that a kinase other than MST may phosphorylate the hydrophobic motif of Lats kinases upon PKA activation. Although unlikely, we cannot exclude the possibility that a residual amount of MST kinase activity is sufficient to activate Lats in response to cAMP. Moreover, it is also possible that PKA may promote Lats phosphorylation by inhibiting a phosphatase.

PKA phosphorylates proteins containing the RRXS/T consensus sequence, and several components of the Hippo pathway with the RRXS/T motif might be direct targets of PKA. Neurofibromin 2 (NF2, also known as merlin), a tumor suppressor and an upstream component of the Hippo pathway (McCartney et al. 2000; Hamaratoglu et al. 2006; Benhamouche et al. 2010; Zhang et al. 2010), has been shown as a direct target of PKA (Alfthan et al. 2004). Based on our data, NF2 is not critical for PKA to induce YAP phosphorylation because the MDA-MB-231 cells have a homozygous NF2 mutation (Dupont et al. 2011), while YAP phosphorylation is properly regulated by cAMP. PKA can also phosphorylate mouse Lats2 at S171 and S362 following forskolin treatment, with the S171 site conserved in Lats1 and warts (Drosophila Lats ortholog). However, mutation of S171 or S362 of mouse Lats2 cannot block forskolin-induced Lats2 activation (FX Yu and KL Guan, unpubl.), indicating that Lats $1 / 2$ are unlikely to be direct targets of PKA responsible for cAMP-induced YAP phosphorylation. Our data are consistent with a model in which Rho functions between PKA and Lats1/2 kinases (Fig. 7).

RhoA regulates the Hippo pathway by modulating the actin cytoskeleton. Formation of actin filaments or generation of cellular tension results in YAP dephosphorylation, nuclear localization, and activation (Dupont et al. 2011; Fernandez et al. 2011; Sansores-Garcia et al. 2011; Wada et al. 2011; Mo et al. 2012; Yu et al. 2012b; Zhao et al. 2012). In addition to RhoA, other Rho family members, such as Rac and Cdc42, can also regulate the Hippo pathway kinases (Zhao et al. 2012). Therefore, the effect of PKA on the Hippo pathway may not be solely mediated by RhoA. Other Rho GTPases or their effectors may participate in the signaling pathway from PKA to Lats (Fig. 7). In support of this, PKA has been shown to phosphorylate PAK (Howe and Juliano 2000), which in principle can lead to rearrangements of the actin cytoskeleton. 
A
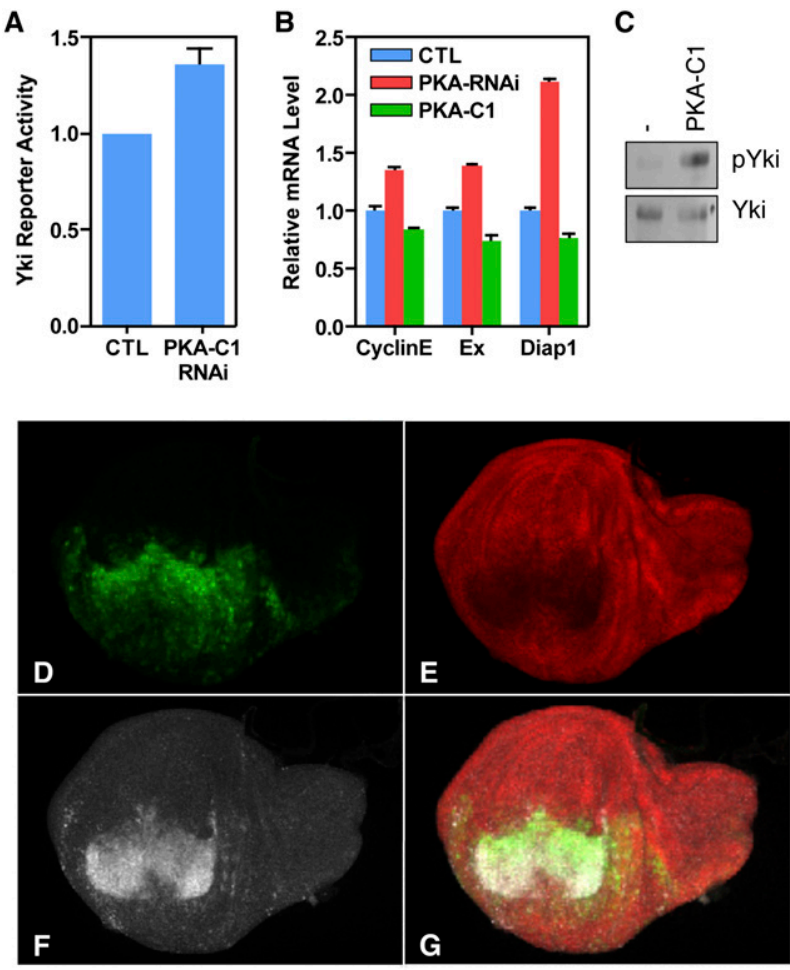

Figure 6. PKA inhibits Yki in Drosophila. (A) In Drosophila $\mathrm{S} \mathrm{R}^{+}$cells, knockdown of PKA-C1 by RNAi increased Yki/Sd reporter activity. $(B)$ Relative transcript levels of $e x, C y c E$, and Diap1 genes in wild-type (blue), C5-Gal4/UAS-PKA-C1 RNAi (red), and C5-Gal4/UAS-PKA-C1 (green) larval wing discs. $(C)$ Yki phosphorylation was increased in C5-Gal4/UAS-PKA-C1 larval wing discs. $(D-F)$ en-Gal4/UAS-PKA-C1 UAS-GFP larval wing discs exhibiting expression of GFP marker ( $D$, green), Diap1 protein $(E$, red), and Caspase3 $(F$, white). $(G)$ Merge of $D-F$.

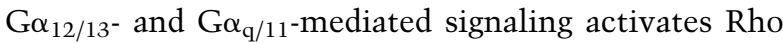
GTPases and YAP (Yu et al. 2012b). The importance of Rho GTPases in PKA-mediated YAP inactivation suggests that $\mathrm{G} \alpha_{\mathrm{s}}$-mediated signals antagonize with $\mathrm{G} \alpha_{12 / 13^{-}}$ and $\mathrm{G} \alpha_{\mathrm{q} / 11}$-mediated signals on the activity of Rho GTPases, which in turn results in induction or repression of YAP phosphorylation. Therefore, differential regulations of Rho GTPases by numerous extracellular molecules will fine-tune the activity of the Hippo pathway and determine cellular responses such as cell proliferation, apoptosis, and differentiation (Fig. 7). This also provides a mechanism of signal integration when cells have to respond to a wide range of extracellular signals.

\section{YAP/TAZ inhibition mediates cellular functions of PKA}

PKA is the first protein kinase purified and is involved in a wide range of physiological regulations (Taylor et al. 2012). This study indicates that inhibition of YAP/TAZ contributes to the physiological function of cAMP or PKA. For example, cAMP can promote adipocyte differentiation, and this process is dependent on inhibition of YAP and TAZ (Fig. 5). Besides adipocyte differentiation, PKA has been shown to induce neuronal differentiation and inhibit osteoblast differentiation (Ravni et al. 2006; Yang et al. 2008). One may speculate that YAP/TAZ play a role in neurogenesis or osteogenesis in response to cAMP signals. Consistent with this notion, YAP or TAZ has been shown to promote osteogenesis and inhibit neuronal differentiation (Hong et al. 2005; Cao et al. 2008; Zhang et al. 2012). Interestingly, RhoA has functions similar to those of YAP/TAZ during various cell differentiation processes (McBeath et al. 2004). Therefore, inhibition of Rho GTPases and YAP/TAZ may serve as a common mechanism in PKA-regulated cell differentiation.

PKA exerts a growth inhibitory effect on most cell and tissue types. YAP and TAZ are putative oncoproteins, and their activation stimulates cell proliferation and inhibits apoptosis. Therefore, PKA may inhibit cell growth by inactivating YAP/TAZ. This notion is supported by the functional analyses in Drosophila in which PKA inhibits the expression of cyclin E and Diap1. Based on the data presented in this study, we propose that inhibition of YAP/TAZ plays a key role in mediating the growth inhibitory effect of PKA. YAP/TAZ activation by either increased protein expression or reduced phosphorylation is associated with a large number of human cancers (Chan et al. 2008; Steinhardt et al. 2008). Many pharmaceutical drugs directly target cellular cAMP levels. We speculate that elevation of cAMP by either PDE inhibitors or adenylate cyclase activators may suppress tumor growth, particularly for those with high activity of YAP or TAZ.

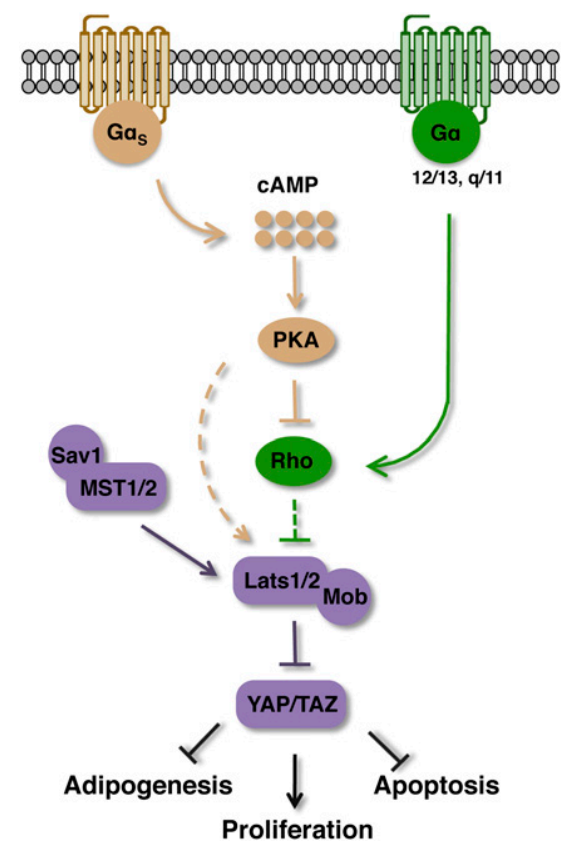

Figure 7. Regulation of the Hippo pathway by cAMP-PKA signaling. Upon stimulation of $\mathrm{G}_{\mathrm{s}}$-coupled GPCR, activation of PKA by cAMP leads to inhibition of Rho GTPases, which indirectly inhibit Lats kinase activity. Stimulation of $\mathrm{G} \alpha_{12 / 13^{-}}$or

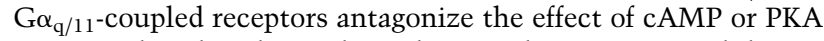
on YAP phosphorylation by inducing Rho GTPases. Inhibition of YAP and TAZ mediates functions of cAMP and PKA on adipogenesis, cell proliferation, and apoptosis. 
Yu et al.

\section{Materials and methods}

Cell culture

MDA-MB-231 cells were cultured in DMEM/F12 medium (Invitrogen). HEK239A, HEK293T, U2OS, and MEFs were cultured in DMEM medium (Hyclone). Primary hepatocytes were isolated from 12-wk-old male mice using a standard protocol and incubated in DMEM medium. All of the above cells were supplemented with $10 \%$ fetal bovine serum (FBS) (Omega Scientific) and $50 \mu \mathrm{g} / \mathrm{mL}$ penicillin/streptomycin $(\mathrm{P} / \mathrm{S})$. MCF10A cells were cultured in DMEM/F12 supplemented with $5 \%$ horse serum (Invitrogen), $20 \mathrm{ng} / \mathrm{mL}$ EGF, $0.5 \mu \mathrm{g} / \mathrm{mL}$ hydrocortisone, $10 \mu \mathrm{g} /$ $\mathrm{mL}$ insulin, $100 \mathrm{ng} / \mathrm{mL}$ cholera toxin, and $50 \mu \mathrm{g} / \mathrm{mL} \mathrm{P} / \mathrm{S}$. For serum starvation, cells were incubated in DMEM or DMEM/F12 without supplements. All cell lines were maintained at $37^{\circ} \mathrm{C}$ with $5 \% \mathrm{CO}_{2}$.

\section{Chemicals}

Epinephrine, glucagon, dexamethasone, troglitazone, and rolipram were purchased from Sigma-Aldrich. IBMX, forskolin, KT5720, ibudilast, and theophylline were purchased from Tocris.

\section{Transfection}

Cells were transfected with plasmid DNA using PolyJet DNA in vitro transfection reagent (Signagen Laboratories) according to the manufacturer's instructions. Dr. Mark Ginsberg (University of California at San Diego) generously provided the GFP-GDI plasmid. The pCMV-Flag-YAP, pCDNA3-MST2 K/R, and pCDNA3-Lats2 $\mathrm{K} / \mathrm{R}$ plasmids have been described elsewhere (Zhao et al. 2010, 2012). RhoA, the PKA catalytic subunit, and Rap1b were in a pCDNA3 vector. The PKA regulatory subunit mutants $(\mathrm{I} \alpha$ and $\mathrm{II} \alpha)$ were in a pEGFP-C1 vector.

\section{RNAi}

Smartpool siRNAs were purchased from Dharmacon, and siRNAs were delivered into cells using RNAiMAX (Invitrogen) according to the manufacturer's instructions. Lentiviral shRNAs in the pLKO.1 vector were purchased from Sigma-Aldrich, and virus was made in HEK293T cells using pMD2.g and PsPAX2 as packaging plasmids. Virus was filtered and used to infect targeting cells. The The RNAi Consortium (TRC) IDs for shRNAs used for the PKA catalytic subunit (PRKACA) are TRCN0000001372 and TRCN0000001373.

\section{Immunoblotting}

Immunoblotting was performed using a standard protocol. Antibodies for pYAP (S127), YAP, TAZ (V386), pCREB, CREB, pMLC2 (S19), pMLC2 (T18/S19), MST1, MST2, and Lats1 were from Cell Signaling Technology. The Lats2 antibody was from Bethyl Laboratories. The HA-HRP, GFP, and MLC2 antibodies were from Santa Cruz Biotechnology. Tubulin, HSP90, and FlagHRP were purchased from Sigma-Aldrich. The PKA antibody was obtained from BD Biosciences. The GAPDH antibody was a gift from Dr. Yan Luo. The Yki antibody was a gift from Dr. Kenneth Irvine. The phos-tag reagents were purchased from Wako Chemicals, and gels containing phos-tag and $\mathrm{MnCl}_{2}$ were prepared according to the manufacturer's instructions. YAP proteins can be separated into multiple bands on phos-tag gels, with the phosphorylated form of YAP proteins migrating at a slower speed.
Immunoprecipitation and Lats kinase assay

Cells were lysed using mild lysis buffer $(50 \mathrm{mM}$ HEPES at $\mathrm{pH}$ $7.5,150 \mathrm{mM} \mathrm{NaCl}, 1 \mathrm{mM}$ EDTA, $1 \%$ NP-40, $10 \mathrm{mM}$ pyrophosphate, $10 \mathrm{mM}$ glycerophosphate, $50 \mathrm{mM} \mathrm{NaF}$, $1.5 \mathrm{mM} \mathrm{Na}_{3} \mathrm{VO}_{4}$, protease inhibitor cocktail [Roche], $1 \mathrm{mM}$ PMSF). Cell lysates were cleared and used for immunoprecipitation. The Lats1 antibody (Cell Signaling Technology) was mixed with cell lysates for $1 \mathrm{~h}$ at $4^{\circ} \mathrm{C}$, and then protein A agarose beads were added in for $1 \mathrm{~h}$. After four washes with lysis buffer, beads were washed once with wash buffer (40 mM HEPES, $200 \mathrm{mM}$ $\mathrm{NaCl}$ ) and once with kinase assay buffer (30 mM HEPES, 50 $\mathrm{mM}$ potassium acetate, $5 \mathrm{mM} \mathrm{MgCl}_{2}$ ). The immunoprecipitated Lats 1 was then subjected to a kinase assay in the presence of $500 \mu \mathrm{M}$ cold ATP, $10 \mu \mathrm{Ci}\left[\gamma^{-}{ }^{32} \mathrm{P}\right] \mathrm{ATP}$, and $1 \mu \mathrm{g}$ of GST-YAP expressed and purified from Escherichia coli as substrate. The reaction mixtures were incubated for $30 \mathrm{~min}$ at $30^{\circ} \mathrm{C}$, terminated with SDS sample buffer, and subjected to SDS-PAGE and immunoblotting.

\section{RNA extraction, reverse transcription, and real-time PCR}

Following forskolin treatments or adipogenesis, cells were washed with cold phosphate-buffered saline (PBS) and subjected to RNA extraction using an RNeasy Plus minikit (Qiagen). RNA samples $(1 \mu \mathrm{g})$ were reverse-transcribed to complementary DNA (cDNA) using iScript reverse transcriptase (Bio-Rad). After dilution, cDNA levels were quantified by real-time PCR using KAPA SYBR FAST quantitative PCR master mix (Kapa Biosystems) and the 7300 real-time PCR system (Applied Biosystems). The primer pairs ( $\mathrm{h}$ and $\mathrm{m}$ indicate human and mouse, respectively) used in this study were $\beta$-actin (h), GCCGACAGGATGCAGAAGGAG ATCA/AAGCATTTGCGGTGGACGATGGA; CTGF (h), CCAA TGACAACGCCTCCTG/TGGTGCAGCCAGAAAGCTC; HPRT (m), GCAGTACAGCCCCAAAATGG/ACAAAGTCCGGCCTG TATCCAA; C/EBP $\alpha(\mathrm{m})$, GCAAAGCCAAGAAGTCGGTGGA/ CCTTCTGTTGCGTCTCCACGTT; PPAR $\gamma(\mathrm{m})$, CTGTCGGTTT CAGAAGTGCCT/CCCAAACCTGATGGCATTGTGAGACA; Adiposin (m), TCCGCCCCTGAACCCTACAA/TAATGGTGAC TACCCCGTCA; FABP4 (m), CGATGAAATCACCGCAGAC GA/AGTCACGCCTTTCATAACACA; and Adoponectin (m), AGATGGCACTCCTGGAGAGAAG/ACATAAGCGGCTTCT CCAGGCT.

\section{Adipogenesis}

Murine 3T3-L1 cells were maintained in DMEM medium containing $10 \%$ calf serum (Hyclone). To initiate adipocyte differentiation, confluent 3T3-L1 cells were switched into FBS-containing DMEM medium. In addition, insulin, dexamethasone, and troglitazone (Tro) were added. In selected samples, IBMX $(250 \mu \mathrm{M})$ or forskolin $(100 \mu \mathrm{M})$ was used to increase cAMP and PKA activity. For experiments using KT5720, 3T3-L1 cells were pretreated with KT5720 $(5 \mu \mathrm{M})$ overnight in advance, and fresh KT5720 was added when adipogenesis was initiated. Two days later, the medium was changed into DMEM containing $10 \%$ FBS and insulin. After another $2 \mathrm{~d}$, cells were incubated in DMEM with FBS. Cells were typically harvested on day 6 depending on the formation and maturation of lipid droplets. Cells were then subjected to RNA extraction or oil red (Sigma-Aldrich) staining according to the manufacturer's instructions.

\section{Luciferase assay}

$\mathrm{S} \mathrm{R}^{+}$cells were cultured in 24-well plates at standard conditions. PKA-C1 was down-regulated by dsRNAs. All the samples were cotransfected with $10 \mathrm{ng}$ of the copia-Renilla luciferase reporter 
as a normalization control and $200 \mathrm{ng}$ of 3xSd_luc (gift from Dr. Jin Jiang) firefly luciferase reporter using Cellfectin II (Invitrogen). Fifty nanograms each of pUAST-Yki, pUAST-HA-sd, and pAc-Gal4 was used in each well to promote the firefly luciferase expression. Luciferase activity was measured after $48 \mathrm{~h}$ of incubation using the Dual-Glo luciferase assay kit (Promega) according to the manufacturer's protocol.

Drosophila stocks, quantitative RT-PCR, and immunocytochemistry

All fruit flies were maintained under standard conditions. Fly stocks UAS-PKA-C1 (ID \#35554) and UAS-PKA-C1-RNAi (ID \#31277) were obtained from the Bloomington Drosophila Stock Center. Total mRNA from Drosophila late third-instar larval wing discs was isolated using the Qiagen RNAeasy kit (Qiagen), and mRNA was reverse-transcribed using Quanta qScriptcDNAsuperMix (Qiagen). Real-time PCR was performed using PerfeCTA SYBR Green FastMix (Qiagen), and data were collected via the Applied Biosystem StepOnePlus real-time PCR system (Life Technologies). The relative amount of specific mRNAs under each condition was calculated after normalization to the Histone 3 transcripts. A standard procedure for immunocytochemistry was followed in this study. Wing discs from late third-instar larvae were dissected in cold PBS and fixed in 4\% PFA for $30 \mathrm{~min}$ at room temperature. Tissues were incubated in primary antibody overnight at $4^{\circ} \mathrm{C}$, followed by $2 \mathrm{~h}$ of secondary antibody incubation at room temperature. Primary antibodies anti-Diap1 mouse (1:200; gift from Dr. Bruce Hay) and anti-Caspase-3 rabbit (1:200; Cell Signaling Technology) were used in this study. Images were collected with an Olympus Fluoview 1000 confocal laser-scanning microscope.

\section{Acknowledgments}

We thank Dr. Rui Gong, Dr. Jung-Soon Mo, Dr. Frank Yuliang $\mathrm{Ma}$, and Dr. Malik Keshwani for comments and technical help. We also thank the Bloomington Drosophila Stock Center for fly stocks, and Dr. Ken Irvine for reagents. J.L.J. is supported by a National Cancer Institute Grant and The Hartwell Foundation. This work is supported by grants from the NIH and CIRM to K.L.G.

\section{References}

Alfthan K, Heiska L, Gronholm M, Renkema GH, Carpen O. 2004. Cyclic AMP-dependent protein kinase phosphorylates merlin at serine 518 independently of p21-activated kinase and promotes merlin-ezrin heterodimerization. J Biol Chem 279: 18559-18566.

Benhamouche S, Curto M, Saotome I, Gladden AB, Liu CH, Giovannini M, McClatchey AI. 2010. Nf2/Merlin controls progenitor homeostasis and tumorigenesis in the liver. Genes Dev 24: 1718-1730.

Callus BA, Verhagen AM, Vaux DL. 2006. Association of mammalian sterile twenty kinases, Mst1 and Mst2, with hSalvador via C-terminal coiled-coil domains, leads to its stabilization and phosphorylation. FEBS J 273: 4264-4276.

Cao X, Pfaff SL, Gage FH. 2008. YAP regulates neural progenitor cell number via the TEA domain transcription factor. Genes Dev 22: 3320-3334.

Chan EH, Nousiainen M, Chalamalasetty RB, Schafer A, Nigg EA, Sillje HH. 2005. The Ste20-like kinase Mst2 activates the human large tumor suppressor kinase Lats1. Oncogene 24: 2076-2086.

Chan SW, Lim CJ, Guo K, Ng CP, Lee I, Hunziker W, Zeng Q, Hong W. 2008. A role for TAZ in migration, invasion, and tumorigenesis of breast cancer cells. Cancer Res 68: 2592-2598.
Cho-Chung YS. 1990. Role of cyclic AMP receptor proteins in growth, differentiation, and suppression of malignancy: New approaches to therapy. Cancer Res 50: 7093-7100.

Dong J, Feldmann G, Huang J, Wu S, Zhang N, Comerford SA, Gayyed MF, Anders RA, Maitra A, Pan D. 2007. Elucidation of a universal size-control mechanism in Drosophila and mammals. Cell 130: 1120-1133.

Dupont S, Morsut L, Aragona M, Enzo E, Giulitti S, Cordenonsi M, Zanconato F, Le Digabel J, Forcato M, Bicciato S, et al. 2011. Role of YAP/TAZ in mechanotransduction. Nature 474: 179-183.

Fernandez BG, Gaspar P, Bras-Pereira C, Jezowska B, Rebelo SR, Janody F. 2011. Actin-capping protein and the Hippo pathway regulate F-actin and tissue growth in Drosophila. Development 138: 2337-2346.

Gloerich M, Bos JL. 2010. Epac: Defining a new mechanism for cAMP action. Annu Rev Pharmacol Toxicol 50: 355-375.

Goulev Y, Fauny JD, Gonzalez-Marti B, Flagiello D, Silber J, Zider A. 2008. SCALLOPED interacts with YORKIE, the nuclear effector of the hippo tumor-suppressor pathway in Drosophila. Curr Biol 18: 435-441.

Hamaratoglu F, Willecke M, Kango-Singh M, Nolo R, Hyun E, Tao C, Jafar-Nejad H, Halder G. 2006. The tumour-suppressor genes NF2/Merlin and Expanded act through Hippo signalling to regulate cell proliferation and apoptosis. Nat Cell Biol 8: 27-36.

Harvey KF, Pfleger CM, Hariharan IK. 2003. The Drosophila Mst ortholog, hippo, restricts growth and cell proliferation and promotes apoptosis. Cell 114: 457-467.

Hong JH, Hwang ES, McManus MT, Amsterdam A, Tian Y, Kalmukova R, Mueller E, Benjamin T, Spiegelman BM, Sharp PA, et al. 2005. TAZ, a transcriptional modulator of mesenchymal stem cell differentiation. Science 309: 1074-1078.

Howe AK, Juliano RL. 2000. Regulation of anchorage-dependent signal transduction by protein kinase A and p21-activated kinase. Nat Cell Biol 2: 593-600.

Huang J, Wu S, Barrera J, Matthews K, Pan D. 2005. The Hippo signaling pathway coordinately regulates cell proliferation and apoptosis by inactivating Yorkie, the Drosophila homolog of YAP. Cell 122: 421-434.

Jia J, Zhang W, Wang B, Trinko R, Jiang J. 2003. The Drosophila Ste20 family kinase dMST functions as a tumor suppressor by restricting cell proliferation and promoting apoptosis. Genes Dev 17: 2514-2519.

Jiang J, Struhl G. 1995. Protein kinase A and hedgehog signaling in Drosophila limb development. Cell 80: 563-572.

Justice RW, Zilian O, Woods DF, Noll M, Bryant PJ. 1995. The Drosophila tumor suppressor gene warts encodes a homolog of human myotonic dystrophy kinase and is required for the control of cell shape and proliferation. Genes Dev 9: 534 546.

Kanai F, Marignani PA, Sarbassova D, Yagi R, Hall RA, Donowitz M, Hisaminato A, Fujiwara T, Ito Y, Cantley LC, et al. 2000. TAZ: A novel transcriptional co-activator regulated by interactions with 14-3-3 and PDZ domain proteins. EMBO J 19: 6778-6791.

Kango-Singh M, Nolo R, Tao C, Verstreken P, Hiesinger PR, Bellen HJ, Halder G. 2002. Shar-pei mediates cell proliferation arrest during imaginal disc growth in Drosophila. Development 129: 5719-5730.

Lai ZC, Wei X, Shimizu T, Ramos E, Rohrbaugh M, Nikolaidis N, Ho LL, Li Y. 2005. Control of cell proliferation and apoptosis by mob as tumor suppressor, mats. Cell 120: 675-685.

Lane ME, Kalderon D. 1993. Genetic investigation of cAMPdependent protein kinase function in Drosophila development. Genes Dev 7: 1229-1243. 
Yu et al.

Lei QY, Zhang H, Zhao B, Zha ZY, Bai F, Pei XH, Zhao S, Xiong Y, Guan KL. 2008. TAZ promotes cell proliferation and epithelialmesenchymal transition and is inhibited by the hippo pathway. Mol Cell Biol 28: 2426-2436.

Lepage T, Cohen SM, Diaz-Benjumea FJ, Parkhurst SM. 1995. Signal transduction by cAMP-dependent protein kinase A in Drosophila limb patterning. Nature 373: 711-715.

Li W, Ohlmeyer JT, Lane ME, Kalderon D. 1995. Function of protein kinase A in hedgehog signal transduction and Drosophila imaginal disc development. Cell 80: 553-562.

Liu CY, Zha ZY, Zhou X, Zhang H, Huang W, Zhao D, Li T, Chan SW, Lim CI, Hong W, et al. 2010. The hippo tumor pathway promotes TAZ degradation by phosphorylating a phosphodegron and recruiting the SCF $\beta$-TrCP E3 ligase. I Biol Chem 285: 37159-37169.

McBeath R, Pirone DM, Nelson CM, Bhadriraju K, Chen CS. 2004. Cell shape, cytoskeletal tension, and RhoA regulate stem cell lineage commitment. Dev Cell 6: 483-495.

McCartney BM, Kulikauskas RM, LaJeunesse DR, Fehon RG. 2000. The neurofibromatosis-2 homologue, Merlin, and the tumor suppressor expanded function together in Drosophila to regulate cell proliferation and differentiation. Development 127: 1315-1324.

Meiri D, Greeve MA, Brunet A, Finan D, Wells CD, LaRose J, Rottapel R. 2009. Modulation of Rho guanine exchange factor Lfc activity by protein kinase A-mediated phosphorylation. Mol Cell Biol 29: 5963-5973.

Miller E, Yang J, Deran M, Wu C, Su AI, Bonamy GM, Liu J, Peters EC, Wu X. 2012. Identification of serum-derived sphingosine-1-phosphate as a small molecule regulator of YAP. Chem Biol 19: 955-962.

Mo JS, Yu FX, Gong R, Brown JH, Guan KL. 2012. Regulation of the Hippo-YAP pathway by protease activated receptor PAR. Genes Dev 29: 2138-2143.

Oh H, Irvine KD. 2008. In vivo regulation of Yorkie phosphorylation and localization. Development 135: 1081-1088.

Pan D, Rubin GM. 1995. cAMP-dependent protein kinase and hedgehog act antagonistically in regulating decapentaplegic transcription in Drosophila imaginal discs. Cell 80: 543552.

Pantalacci S, Tapon N, Leopold P. 2003. The Salvador partner Hippo promotes apoptosis and cell-cycle exit in Drosophila. Nat Cell Biol 5: 921-927.

Qiao J, Holian O, Lee BS, Huang F, Zhang J, Lum H. 2008. Phosphorylation of GTP dissociation inhibitor by PKA negatively regulates RhoA. Am I Physiol Cell Physiol 295: C1161-C1168.

Ravni A, Bourgault S, Lebon A, Chan P, Galas L, Fournier A, Vaudry H, Gonzalez B, Eiden LE, Vaudry D. 2006. The neurotrophic effects of PACAP in PC12 cells: Control by multiple transduction pathways. J Neurochem 98: 321-329.

Ren F, Zhang L, Jiang J. 2010. Hippo signaling regulates Yorkie nuclear localization and activity through 14-3-3 dependent and independent mechanisms. Dev Biol 337: 303-312.

Rosen ED, MacDougald OA. 2006. Adipocyte differentiation from the inside out. Nat Rev Mol Cell Biol 7: 885-896.

Sansores-Garcia L, Bossuyt W, Wada K, Yonemura S, Tao C, Sasaki H, Halder G. 2011. Modulating F-actin organization induces organ growth by affecting the Hippo pathway. EMBO I 30: $2325-2335$.

Sassone-Corsi P. 2012. The cyclic AMP pathway. Cold Spring Harb Perspect Biol 4: a011148.

Steinhardt AA, Gayyed MF, Klein AP, Dong J, Maitra A, Pan D, Montgomery EA, Anders RA. 2008. Expression of Yes-associated protein in common solid tumors. Hum Pathol 39: 1582-1589.
Stork PJ, Schmitt JM. 2002. Crosstalk between cAMP and MAP kinase signaling in the regulation of cell proliferation. Trends Cell Biol 12: 258-266.

Tapon N, Harvey KF, Bell DW, Wahrer DC, Schiripo TA, Haber DA, Hariharan IK. 2002. salvador promotes both cell cycle exit and apoptosis in Drosophila and is mutated in human cancer cell lines. Cell 110: 467-478.

Taylor SS, Ilouz R, Zhang P, Kornev AP. 2012. Assembly of allosteric macromolecular switches: Lessons from PKA. Nat Rev Mol Cell Biol 13: 646-658.

Tkachenko E, Sabouri-Ghomi M, Pertz O, Kim C, Gutierrez E, Machacek M, Groisman A, Danuser G, Ginsberg MH. 2011. Protein kinase A governs a RhoA-RhoGDI protrusion-retraction pacemaker in migrating cells. Nat Cell Biol 13: 660-667.

Udan RS, Kango-Singh M, Nolo R, Tao C, Halder G. 2003. Hippo promotes proliferation arrest and apoptosis in the Salvador/ Warts pathway. Nat Cell Biol 5: 914-920.

Wada K, Itoga K, Okano T, Yonemura S, Sasaki H. 2011. Hippo pathway regulation by cell morphology and stress fibers. Development 138: 3907-3914.

Wu S, Huang J, Dong J, Pan D. 2003. hippo encodes a Ste-20 family protein kinase that restricts cell proliferation and promotes apoptosis in conjunction with salvador and warts. Cell 114: 445-456.

Wu S, Liu Y, Zheng Y, Dong J, Pan D. 2008. The TEAD/TEF family protein Scalloped mediates transcriptional output of the Hippo growth-regulatory pathway. Dev Cell 14: 388-398.

Xu T, Wang W, Zhang S, Stewart RA, Yu W. 1995. Identifying tumor suppressors in genetic mosaics: The Drosophila lats gene encodes a putative protein kinase. Development 121: 1053-1063.

Yang DC, Tsay HJ, Lin SY, Chiou SH, Li MJ, Chang TJ, Hung SC. 2008. cAMP/PKA regulates osteogenesis, adipogenesis and ratio of RANKL/OPG mRNA expression in mesenchymal stem cells by suppressing leptin. PLOS ONE 3: e1540.

Yu FX, Guan KL. 2013. The Hippo pathway: Regulators and regulations. Genes Dev 27: 355-371.

Yu FX, Mo JS, Guan KL. 2012a. Upstream regulators of the Hippo pathway. Cell Cycle 11: 4097-4098.

Yu FX, Zhao B, Panupinthu N, Jewell JL, Lian I, Wang LH, Zhao J, Yuan $\mathrm{H}$, Tumaneng $\mathrm{K}$, Li H, et al. 2012b. Regulation of the Hippo-YAP pathway by G-protein-coupled receptor signaling. Cell 150: 780-791.

Zhang L, Ren F, Zhang Q, Chen Y, Wang B, Jiang J. 2008. The TEAD/TEF family of transcription factor Scalloped mediates Hippo signaling in organ size control. Dev Cell 14: 377-387.

Zhang N, Bai H, David KK, Dong J, Zheng Y, Cai J, Giovannini M, Liu P, Anders RA, Pan D. 2010. The Merlin/NF2 tumor suppressor functions through the YAP oncoprotein to regulate tissue homeostasis in mammals. Dev Cell 19: 27-38.

Zhang H, Deo M, Thompson RC, Uhler MD, Turner DL. 2012. Negative regulation of Yap during neuronal differentiation. Dev Biol 361: 103-115.

Zhao B, Wei X, Li W, Udan RS, Yang Q, Kim J, Xie J, Ikenoue T, $\mathrm{Yu}$ J, Li L, et al. 2007. Inactivation of YAP oncoprotein by the Hippo pathway is involved in cell contact inhibition and tissue growth control. Genes Dev 21: 2747-2761.

Zhao B, Ye X, Yu J, Li L, Li W, Li S, Yu J, Lin JD, Wang CY, Chinnaiyan AM, et al. 2008. TEAD mediates YAP-dependent gene induction and growth control. Genes Dev 22: 1962-1971.

Zhao B, Li L, Tumaneng K, Wang CY, Guan KL. 2010. A coordinated phosphorylation by Lats and CK1 regulates YAP stability through SCF( $\beta$-TRCP). Genes Dev 24: 72-85.

Zhao B, Li L, Wang L, Wang CY, Yu J, Guan KL. 2012. Cell detachment activates the Hippo pathway via cytoskeleton reorganization to induce anoikis. Genes Dev 26: 54-68. 


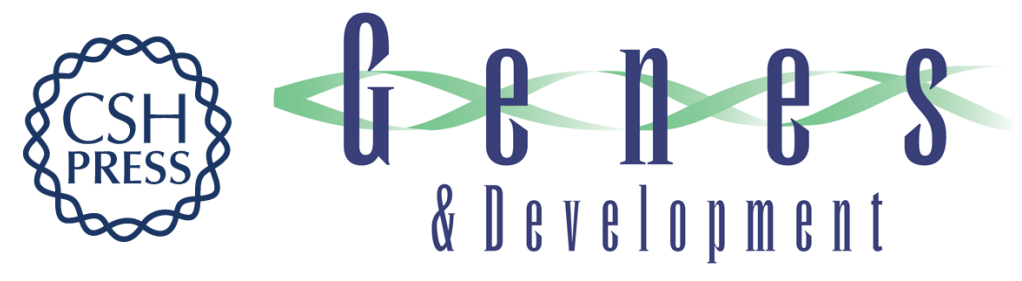

\section{Protein kinase A activates the Hippo pathway to modulate cell proliferation and differentiation}

Fa-Xing Yu, Yifan Zhang, Hyun Woo Park, et al.

Genes Dev. 2013, 27:

Access the most recent version at doi:10.1101/gad.219402.113

\section{Supplemental http://genesdev.cshlp.org/content/suppl/2013/06/10/27.11.1223.DC1 Material \\ Related Content \\ PKA Augments Hippo Signaling \\ Annalisa M. VanHook \\ Sci. Signal. June , 2013 6: ec138 \\ References This article cites 63 articles, 24 of which can be accessed free at: \\ http://genesdev.cshlp.org/content/27/11/1223.full.html\#ref-list-1 \\ Articles cited in: \\ http://genesdev.cshlp.org/content/27/11/1223.full.html\#related-urls \\ License \\ Email Alerting \\ Receive free email alerts when new articles cite this article - sign up in the box at the top \\ Service right corner of the article or click here.}

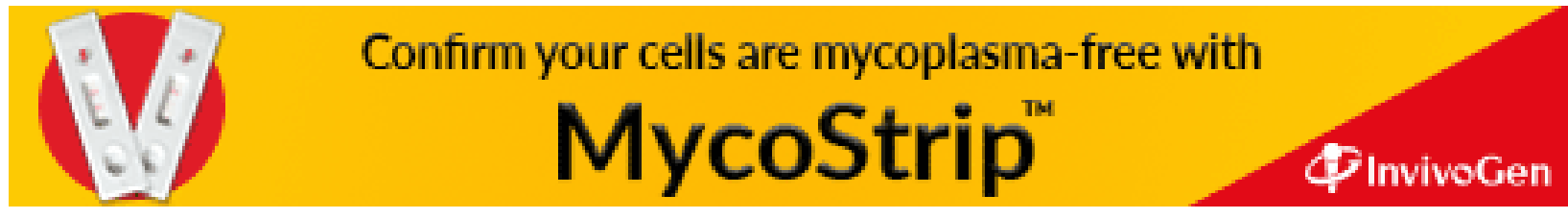

